\title{
HLA-DR15 Antigen
}

National Cancer Institute

\section{Source}

National Cancer Institute. HLA-DR15 Antigen. NCI Thesaurus. Code C105974.

HLA-DR15 antigen is a set of polymorphic glycoprotein complexes encoded by the human HLA-DRA and HLA-DRB1 genes. This protein complex is involved in the presentation of antigens to CD4+ T lymphocytes. 